\title{
Perceptions and Practicality of Combating Wheat Based 2050 Food Security Challenges
}

ISSN: 2637-7659

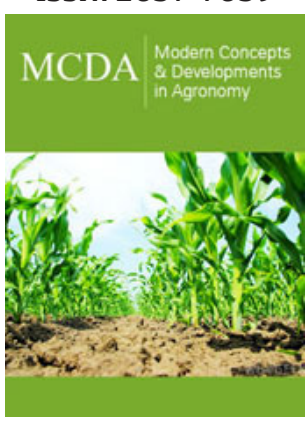

*Corresponding author: Mujeeb-Kazi A Texas A\&M Agrilife Research \& Extension Center, Amarillo, Texas, USA

Submission: 誨 July 13, 2020

Published: 侽July 17, 2020

Volume 6 - Issue 5

How to cite this article: Mujeeb-Kazi A, Niaz Ali, Mahjabeen Tariq, Rumana Keyani. Perceptions and Practicality of Combating Wheat Based 2050 Food Security Challenges. Mod Concep Dev Agrono. 6(5). MCDA. 000649. 2020. DOI: 10.31031/MCDA.2020.06.000649

Copyright@Mujeeb-Kazi A, This article is distributed under the terms of the Creative Commons Attribution 4.0 International License, which permits unrestricted use and redistribution provided that the original author and source are credited.

\author{
Mujeeb-Kazi A ${ }^{1 *}$, Niaz Alï ${ }^{2}$, Mahjabeen Tariq ${ }^{3}$ and Rumana Keyani ${ }^{4}$ \\ ${ }^{1}$ Texas A\&M University, Texas, USA \\ ${ }^{2}$ Hazara University, Manshera, Pakistan \\ ${ }^{3}$ Arid Agriculture University, Rawalpindi, Pakistan \\ ${ }^{4}$ COMSATS University, Islamabad, Pakistan
}

Opinion

\section{The Foundation}

Wheat has been unequivocally recognized as the major conduit to combat food security challenges envisioned for 2050 when the global populace is approximated to touch about 9.7 billion. Paramount for meeting annual wheat yield increases projected at $1.7 \%$ have to be ways and means that deliver swift progressive enhancement numbers, that have been inconsistent so far. No matter what advancements occur in cutting edge science, if those outputs are not swiftly translated into tons per hectare via exploiting smooth multidisciplinary integrated crop improvement protocols, the desired applied targets will remain elusive. The enormous advances in molecular technology and the recent range of global quality publications offer us great impetus that the set targets covering food security, zero hunger, sustainability development goals shall be achieved. However, it is crucial that a unified research effort exists and operates in tandem either at one location or structured around partners that are target driven as dictated by the 2050 timed output constraints driven by climate change.

In essence, progress will be seen by integrating disciplines that execute the development program hand in hand via systematic crop breeding filial generation advance, where at each stage the technologies used permit efficient selective plant generation advance. Such a well integrated multidisciplinary wheat improvement program is a dire need as only then shall we be able to see outputs that hopefully deliver gains in a timely manner. Have yields plateud? How can this bottle neck be removed? No matter what the constraints, the ultimate measure of research progress will be yield enhancement on cultivation grounds that themselves will get limited versus time. When grounds become limiting, moving forward is a huge hurdle. A lot has been done using what had been available to give us the green revolution whereby major biotic and abiotic stresses were well addressed. Quantum increases in productivity emerged with experimental creativity in the mid-1960's. A major innovative effort of the mid-1970's was when winter/spring wheats were crossed generating the famous T1BL.1RS wheat/rye translocation wheats that spread far and wide, enhancing yield levels across the globe where these wheats were adapted. Stagnation since then necessitated that strategies to widen the genetic base were identified and exploited. Using novel genetic components; the initial approach was pursued in 1876 and extended into 1900's. These initial academic investigations with limited success subsequently paved the way for a surge in experimentation in few global research centers and by early 1980's "wheat wide crosses" had gained prominence. The basic success in wide hybridization countering cross incompatibility barriers was achieved and fortuitously limited practical gains emerged from a few hybrid combinations of which the 
most significant were materials that were superior in germplasm performance [1].

These have been reported and reviewed in contributions of [2-8]. Missing was the need of a greater range of genetic diversity that could be exploited swiftly and cover a wide range of stress factors that are now linked with the hazards of climate change that penalize optimum food security projections [9]. Aegilops tauschii or goat grass emerged as the answer and during 1987 until 2004 numerous genetic stocks called synthetic wheats were produced that offered from the over 1000 entries produced in few global locations, an arsenal of genes that are a gold mine of diversity capable of combatting food production constraints adequately. These genetic stocks had resistances and tolerances to almost all biotic/abiotic stresses and became the resource for facilitating molecular tool development [10-12]. The stocks are housed in several gene banks, are freely distributed and show great promise to be able to address wheat production attributes perfectly geared to combat the adversities of climate change. Apart from stress traits these stocks have enhanced yield per se by $22.7 \%$ based upon data gathered from field cultivation over numerous hectares through a cultivar CHAUNMAI 42 derived from a synthetic wheat cross combination [13].

\section{Opinions and perceptions}

Research is vital for generating quality platforms of outputs that is an agglomeration of three major facets encompassing basic, strategic and applied categories. These three facets require a common base where genetic diversity and its wise exploitation is paramount. Wise in the sense that researchers must recognize that experimentation cannot be open ended. The light at the end of the tunnel must be reached on time and impact seen if the demands for food security are well met and the complex environmental hazardous factors are circumvented. We feel that there is currently ample usable diversity generated and globally available that adequately covers the basic/strategic facets of a research program. From the outputs of these two segments stringently focusing on the third "applied" component is the need. The available genetic stock data elucidates with clarity what materials should be selected for applied operations that can swiftly provide returns that mitigate all major environmental hazards jeopardizing food security projections. This is not the time to delve further into basic science but instead astutely move forward towards achieving positive practical outputs by using smart selective strategies that harness the needed genetic diversity stored in gene banks and well catalogued if the annual gains [14] up to $1.7 \%$ are achieved leading us to the I billion tons production by the 2050 food security target. We opine that the smart course would be to thoroughly exploit the over 1000 D genome synthetics that provide the answer of excellent diversity for almost all major stress factors. These stocks are freely available, are user-friendly for breeding, integral to applications of the current molecular technologies and genetically excellent as genetic recombination with bread wheat is at a maximum. Literature is loaded with the resistance/tolerance information of these stocks and except for quality tolerance to aluminum toxicity the expanse of beneficial coverage of its alleles is profound. With this wealth we must ask the question "What is the need to take a big step backward towards basic research instead of focusing on the over 1000 plus synthetics of the D genome that fit the applied scenario and is the answer to deliver swiftly the outputs needed for the 2050 food security mandate". We do not see the need to widen the $\mathrm{D}$ genome diversity as suggested by Mirzaghaderi and Mason [15] as by using other higher ploidy D genome genera the simple diploid level breeding effort will be confounded by the unrelated other genomes present and slow the breeding process.

Our perception is that this abundant $\mathrm{D}$ genome diploid based created diversity in spring and winter habits embraces wide pathogen and environmental stress coverage that governs global wheat productivity performance, has supporting value for mineral toxicity like boron, phytic acid variation, pre-harvest sprouting tolerance, insect/curl mite resistance, possesses novel desirable grain quality attributes, superb DNA polymorphisms used in molecular applications, micronutrient richness, and above all unique yield components essential for elevating productivity returns. This germplasm has the variation that can also enrich the durum wheats across one genome by cytogenetic manipulation and many samples possess multiple stress attributes that can speed up agglomeration of needed attributes. This strength of accessibility to enormous diversity of practical value easily recombined with wheat renders this progenitor genome resource as pivotal for wheat improvement and combat food security is our stance. Integral to this strategy is to speed up varietal development by the appropriately timed underutilized doubled haploid route to shorten the breeding cycle and exploiting its other practical additive values [16].

\section{References}

1. Kishii M (2019) An update of recent use of Aegilops species in wheat breeding. Frontiers in Plant Science 10: 585.

2. Sharma HC (1995) How wide can a wide cross be? Euphytica 82(1): 4364.

3. Mujeeb-Kazi A, Ali N, Ibrahim A, Napar AA, Jamil M, et al. (2017) Tissue culture mediated allelic diversification and genomic enrichment of wheat to combat production constraints and address food security. Plant Tissue Culture and Biotechnology 27(1): 89-140.

4. Mujeeb-Kazi A, Munns R, Rasheed A, Ogbonnaya FC, Ali N, et al. (2019) Breeding strategies for structuring salinity tolerance in wheat. Advances in Agronomy 155: 121-187.

5. Mujeeb-Kazi A, Ali N, Dundas I, Larkin P, Morghonov A, et al. (2020) Way forward perceptions for harnessing genetic diversity to address wheat based food security projections. In: Irwin Goldman (Ed.), Plant Breeding Reviews, (Invited Chapter).

6. Gupta PK (2016) Use of alien genetic variation for wheat improvement. In: Rajpal VR et al (Eds.), Molecular Breeding for Sustainable Crop Improvement. Springer International Publishing, Switzerland, pp. 1-30.

7. Gupta PK, Vasistha NK (2018) Wheat cytogenetics and cytogenomics: the present status. Nucleus 61(3): 195-212. 
8. Gupta PK, Balyan HS, Sharma S, Kumar R (2020) Genetics of yield abiotic stress tolerance and biofortification in wheat. Theor Appl Genet 133(5): 1569-1602.

9. Wani SH, Khan H, Riaz A, Joshi DC, Hussain W, et al. (2020) Recent advances in using genetic diversity for developing climate resilient wheats. Advances in Agronomy.

10. Ogbonnaya FC, Abdalla O, Mujeeb KA, Kazi AG, Xu SS, et al. (2013) Synthetic hexaploid in wheat improvement. In: Janick J (Ed.), Plant Breeding Reviews 37. ( $1^{\text {st }}$ edn), John Wiley \& Sons, Inc., Hoboken, New Jersey, USA, pp. 35-122.

11. Rosyara U, Kishii M, Payne T, Sansaloni SN, Singh RP, et al. (2019) Genetic contribution of synthetic hexaplod wheat to CIMMYT's Spring Bread Wheat Breeding Germplasm. Scientific Reports 9(1): 12355.

12. Zhang H, Mittal N, Leamy LJ, Barazani O, Song BH (2017) Back into the wild-Apply untapped genetic diversity of wild relatives for crop improvement. Evol Appl 10(1): 5-24.
13. Li A, Liu D, Yang W, Kishii M, Mao L, et al. (2018) Synthetuc hexaloid wheat; Yesterday, today and tomorrow. Engineering 4(4): 552-558.

14. Tadesse W, Sanchez GM, Assefa SG, Amri A, Bishaw Z, et al. (2019) Genetic gains in wheat breeding and its role in feeding the world. Crop Breeding Genetics and Genomics 1: e190005.

15. Mirzaghaderi G, Mason AS (2019) Broadening the wheat D genome. Theor Appl Genet 132(5): 1295-1307.

16. Mujeeb-Kazi A, Gul A, Ahmad J, Mirza JI (2006) A simplified and effective protocol for production of bread wheat haploids (n $1 / 43 \times 1 / 421, A B D$ ) with some application areas in wheat improvement. Pak J Bot 38(2): 393-406. 